\title{
Estimation of equivalent sound pressure levels of community noise and road traffic noise
}

\author{
Takumasa Yoshida \\ Department of Architectural Hygiene Engineering and Housing, \\ The Institute of Public Health, \\ 4-6-1 Shirokanedai, Minato-ku, Tokyo, 108 Japan
}

(Received 3 April 1993)

\begin{abstract}
This study was aimed at making sure usefulness of assumption of Weibull distribution or Rayleigh distribution for estimating $L_{\mathrm{eq}}$ of community noise and road traffic noise from noise levels in $L_{5}, L_{50}$ and $L_{95}$. The results showed that Weibull distribution was very useful to estimate $L_{\mathrm{eq}}$ except few cases like the exponential distribution of noise levels and that Rayleigh distribution was also useful with permitting errors of about $1.5 \mathrm{~dB}$ (A) except such cases as distributions of noise levels were largely skewed to lower levels or higher levels.
\end{abstract}

Keywords: Community Noise, Road traffic noise, $L_{\mathrm{eq}}$, Weibull distribution, Rayleigh distribution

PACS number: 43. 50. Lj, 43. 50. Qp

\section{INTRODUCTION}

As for evaluation of community noise and road traffic noise, many noise indices have been used and $L_{\text {eq }}$ (equivalent sound pressure level) and $L_{\alpha}$ (percentile noise level, e.g. $L_{5}, L_{50}, L_{95}$ ) have often been used in Japan. So relations among these indices, especially between $L_{\mathrm{eq}}$ and $L_{\alpha}$, are very important to evaluate road traffic noise and community noise. ${ }^{1)}$ Hence, Yamaguchi et al. have reported that two types of approach are there to investigate into relations among noise indices for road traffic noise. ${ }^{1)}$ One of them is based on probability theory ${ }^{2-4)}$ and the other is only based on measured data. ${ }^{5,6)}$ As for road traffic noise, for example, Takagi et al. ${ }^{3)}$ investigated into noise on assumption of exponential distributions of headway for road traffic flows and Ohta et $a ._{.}{ }^{2)}$ studied into noise in a general distribution of headway for road traffic flows. Ishii and Tachibana ${ }^{6)}$ reported a simple relation between $L_{\mathrm{eq}}$ and $L_{50}$ in relation to a variable of $l / d$, where $l$ was a minimum distance from a road to a receive point of its noise and $d$ was a mean headway. A simple relationship between $L_{\mathrm{eq}}$ and $L_{50}$ in relation to the variable of $l / d$ has been proposed by the Acoustical Society of Japan for estimation of road traffic noise of freely flowing traffic. ${ }^{7)}$ As for community noise, for example, Nishinomiya ${ }^{8)}$ reported that Weibull distribution was useful to estimate community noise. Mori et al. ${ }^{9)}$ also studied into relations between the parameters of Weibull distribution and physical and social parameters of environments for estimating $L_{\text {eq }}$, and reported that the shape parameter of Weibull distribution was near 2.0 in average for community noise.

This suggests that levels of comminity noise fluctuate around Rayleigh distribution, because Rayleigh distribution corresponds to Weibull distribution with the shape parameter of 2.0.10)

This study was aimed at estimating $L_{\text {eq }}$ of community noise and road traffic noise on assumption of Weibull distribution or Rayleigh distribution by only using data of noise levels in $L_{5}, L_{50}$ and $L_{95}$, and at making sure usefulness or limitation of these assumptions of distributions of noise levels. 


\section{WEIBULL DISTRIBUTION AND RAYLEIGH DISTRIBUTION}

The density function and distribution function of Weibull distribution are given by

$$
\begin{gathered}
f(x)=\frac{k \cdot x^{k-1}}{b^{k}} \exp \left(-\frac{x^{k}}{b^{k}}\right) \\
F(x)=1-\exp \left(-\frac{x^{k}}{b^{k}}\right)
\end{gathered}
$$

where $b$ is a scale parameter, $k$ is a shape parameter and $x=L$ (noise levels) - Min. (a minimum noise level). Then, $L_{\alpha}$ is represented as follows. ${ }^{8)}$

$$
L_{\alpha}=b\left(-\ln \alpha^{\prime}\right)^{(1 / k)}+\text { Min. }
$$

where $\alpha^{\prime}=1-F(x)\left(\alpha^{\prime}=\alpha / 100,0<\alpha^{\prime}<1\right)$. If $L_{95}$, $L_{50}$ and $L_{5}$ are known, the parameters of Weibull distribution can be estimated by plotting each of $L_{95}, L_{50}$ and $L_{5}$ in a Weibull probability paper. ${ }^{8)}$ But this is a roughly approximate method with trial and error because of lack of the minimum level ${ }^{9)}$ and it cannot often be known if fitting $L_{95}, L_{50}$ and $L_{5}$ to a probability paper of Weibull distribution is a exact fit. So, the following procedure of calculation was used in this study for estimating the parameters of Weibull distribution.

Now, the following equations are obtained from the Eq. (3).

$$
\begin{aligned}
& \text { Min. }=L_{5}-b(-\ln (.05))^{(1 / k)} \\
& \text { Min. }=L_{50}-b(-\ln (.50))^{(1 / k)} \\
& \text { Min. }=L_{95}-b(-\ln (.95))^{(1 / k)}
\end{aligned}
$$

From the Eqs. of (4-1) (4-3), the following equations are also obtained.

$$
\begin{aligned}
& b=\left(L_{5}-L_{50}\right) /\left\{(-\ln (.05))^{(1 / k)}-(-\ln (.50))^{(1 / k)}\right\} \\
& b=\left(L_{50}-L_{95}\right) /\left\{(-\ln (.50))^{(1 / k)}-(-\ln (.95))^{(1 / k)}\right\}
\end{aligned}
$$

From the Eqs. of (5-1) and (5-2), the following Equation is obtained.

$$
d_{2} e^{K M_{1}}-d_{3} e^{K M_{2}}+d_{1} e^{K M_{3}}=0
$$

where $K=1 / k, M_{1}=\ln (-\ln (.05)), M_{2}=\ln (-\ln (.50))$, $M_{3}=\ln (-\ln (.95)), \quad d_{1}=L_{5}-L_{50}, \quad d_{2}=L_{50}-L_{95}$ and $d_{3}=L_{5}-L_{95}$.

At first, the parameter of $K$ can be calculated by using Newton's method for the Eq. (6). Next, the parameter of $b$ can be obtained by using the Eq. of (5-1) and (5-2). Then, Min. can be obtained by using the Eq. (4-1) (4-3). At last, $L_{\text {eq }}$ can be estimated by the following equation. ${ }^{8)}$

$$
\begin{aligned}
L_{\mathrm{eq}}= & (10 / \ln (10)) \cdot \ln \left\{\int_{0}^{\infty} \exp (-x)\right. \\
& \left.\cdot \exp \left((\ln (10) / 10) b \cdot x^{(1 / k)}\right) d x\right\}+ \text { Min. }
\end{aligned}
$$

The Eq. (7) can be calculated by, for example, Gauss-Laguerre method, ${ }^{8)}$ but it is very complicated.

By the way, the function (1) represents Rayleigh distribution if the shape parameter $k$ is equal to 2 . If $b / \sqrt{2}$ is denoted by $s$, the percentile levels $\left(L_{\alpha}\right)$ are represented by

$$
L_{\alpha}=s \sqrt{2\left\{-\ln \left(\alpha^{\prime}\right)\right\}}+\text { Min. }
$$

Then, $L_{5}, L_{50}, L_{95}$ are directly proportional to the scale parameter of $s$ and these noise indices are represented as follows.

$$
\begin{aligned}
& L_{5} \fallingdotseq 2.45 s+\text { Min. } \\
& L_{50} \fallingdotseq 1.18 s+\text { Min. } \\
& L_{95} \fallingdotseq 0.32 s+\text { Min. }
\end{aligned}
$$

Furthermore, $L_{\mathrm{eq}}$ is represented by the following equation. $^{8)}$

$$
\begin{aligned}
L_{\mathrm{eq}}= & \frac{s^{2}}{2}\left(\frac{\ln 10}{10}\right)+\left(\frac{10}{\ln 10}\right) \\
& \cdot \ln \left(1+s \frac{\ln 10}{10} \sqrt{\pi / 2}\right)+\text { Min. }
\end{aligned}
$$

Hence, if some errors can be permitted within about $1.5 \mathrm{~dB}(\mathrm{~A})$, the following equations are obtained in approximation.

$$
\begin{array}{ll}
L_{\text {eq }} \fallingdotseq 1.60 s+\text { Min. } & (0<s<10) \\
L_{\text {eq }} \fallingdotseq s^{1.27}+\text { Min. } & (0<s<15)
\end{array}
$$

These relationships were obtained by regression

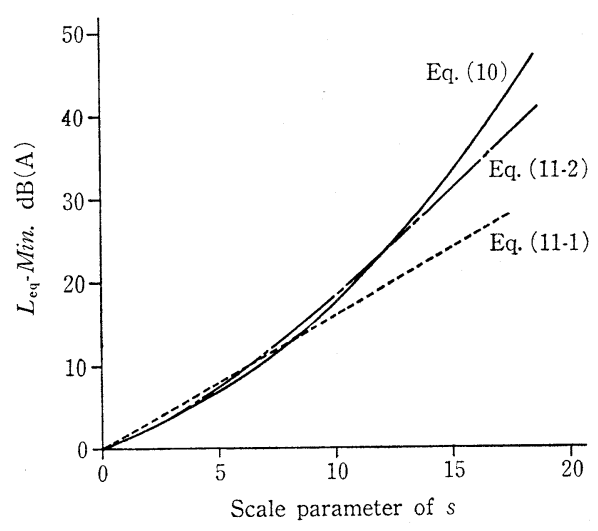

Fig. 1 Differences among the equations of (10), (11-1) and (11-2). 


\section{T. YOSHIDA: ESTIMATION OF $L_{e q}$ OF VARIOUS NOISES}

methods. Figure 1 shows differences among the Eqs. (10), (11-1) and (11-2). Difference between Eq. (10) and Eq. (11-2) becomes larger at $s>15$ and difference between Eq. (10) and Eq. (11-1) becomes larger at $s>10$. If $L_{95}, L_{50}$ and $L_{5}$ are known, the parameter of $s$ and Min. can be estimated by using the following equations which are obtained from the Eqs. of (9-1), (9-2) and (9-3).

$$
\begin{aligned}
& s=(1 / 2)\left\{\frac{L_{5}-L_{50}}{1.27}+\frac{L_{50}-L_{95}}{0.86}\right\} \\
& \text { Min. }=L_{5}-2.45 s
\end{aligned}
$$

Hence, the minimum noise levels estimated by $(9-1)$, (9-2) and (9-3) are often different from one another, because Rayleigh distribution is assumed instead of Weibull distribution. So accuracy of estimating $L_{\mathrm{eq}}$ on assumption of Rayleigh distribution depends on the Eq. of (9-1), (9-2) or (9-3). It was found in preliminary calculations that the Eq. (9-1) led to the best estimation of the minimum levels for estimating $L_{\text {eq }}$, as far as the data in this study. So the Eq. (13) was used in this study for estimat- ing minimum noise levels, and $L_{\mathrm{eq}}$ was calculated by using the Eq. of (10), (11-1) or (11-2).

\section{ESTIMATION OF $\boldsymbol{L}_{\text {eq }}$ FROM $\boldsymbol{L}_{\alpha}$}

Noise 1 involved road traffic noises measured at main roads far from traffic signals or crossings. Noise 2 also involved road traffic noises measured at main roads near traffic signals or crossings. Noise 3 involved road traffic noises of highways. Noise 4 involved environmental noises. Some of them contained aircraft noise, construction noise or railway noise. Each case of the noises measured contained $250 \sim 1,000$ data which were measured in height of 1.2 meters above the ground and were read at intervals of $5 / 3$ seconds. Noise 5 was calculated by some simulations for freely flowing traffic. Noise 6 was calculated by some simulations for traffic flows with signal stops. In these simulations, such parameters were assumed in each case as mean headway between cars, mean speeds of cars, rates of large cars, a minimum distance from a road to a receive point and lane numbers of the road. Each position of

Table 1 Noises using for estimation of $L_{\mathrm{eq}}$.

\begin{tabular}{llccc}
\hline Noises & \multicolumn{1}{c}{ Sources } & $\begin{array}{c}\text { Numbers } \\
\text { of cases }\end{array}$ & $\begin{array}{c}\text { Ranges of } \\
L_{5}-L_{95}\end{array}$ & $\begin{array}{c}\text { Measured or } \\
\text { calculated }\end{array}$ \\
\hline Noise 1 & Roads far from signals & 20 & $6 \sim 23 \mathrm{~dB}(\mathrm{~A})$ & \\
Noise 2 & Roads near signals & 20 & $10 \sim 31$ & Measured \\
Noise 3 & Highways & 12 & $7 \sim 13$ & \\
Noise 4 & Environments & 24 & $5 \sim 34$ & Calculated \\
\hline Noise 5 & Freely flowing road traffics & 80 & $4 \sim 19$ & \\
Noise 6 & Road traffics near a signal & 48 & $8 \sim 34$ & \\
Noise 7 & (Normal distribution) & 30 & $9 \sim 31$ & \\
\hline
\end{tabular}

* Each case of noises measured contained $250 \sim 1,000$ data at intervals of $5 / 3 \mathrm{~s}$ and each case of noises calculated contained 3,000 data calculated at intervals of $1 \mathrm{~s}$.

\begin{tabular}{|c|c|c|c|c|c|c|}
\hline \multirow{2}{*}{ Noise } & \multirow{2}{*}{$\begin{array}{l}\text { Corr. } \\
\text { coef. }\end{array}$} & \multicolumn{3}{|c|}{$\begin{array}{c}\text { Differences of } L_{\mathrm{eq}} \\
\text { (measured) }-(\text { estimated })\end{array}$} & \multicolumn{2}{|c|}{$\begin{array}{c}\text { Parameters of } \\
\text { Weibull distribution }\end{array}$} \\
\hline & & Mean & Ranges & s.e. & $k$ & $b$ \\
\hline Noise 1 & 0.996 & 0.6 & $0.0 \sim 1.3$ & 0.3 & $1.52 \sim 5.09$ & $3.02 \sim 22.26$ \\
\hline Noise 2 & 0.999 & 0.3 & $-0.1 \sim 1.2$ & 0.3 & $1.54 \sim 7.24$ & $4.77 \sim 38.52$ \\
\hline Noise 3 & 0.972 & 0.5 & $0.0 \sim 1.5$ & 0.5 & $1.25 \sim 5.05$ & $2.87 \sim 10.79$ \\
\hline Noise 4 & 0.979 & 0.3 & $-4.3 \sim 3.7$ & 1.3 & $0.71 \sim 1.93$ & $2.20 \sim 10.96$ \\
\hline Noise 5 & 0.998 & 0.2 & $-0.3 \sim 1.0$ & 0.2 & $1.34 \sim 5.28$ & $1.96 \sim 10.44$ \\
\hline Noise 6 & 0.998 & 0.8 & $-0.1 \sim 2.5$ & 0.4 & $1.95 \sim 9.34$ & $5.40 \sim 39.82$ \\
\hline Noise 7 & 0.988 & 0.5 & $-0.2 \sim 1.6$ & 0.4 & $1.70 \sim 3.72$ & $6.39 \sim 15.59$ \\
\hline
\end{tabular}

Table 2 Estimation of $L_{\mathrm{eq}}$ on the assumption of Weibull distribution.

Corr. coef.: correlation coefficients for regressions between $L_{\mathrm{eq}}$ measured and $L_{\mathrm{eq}}$ estimated. 
Table 3 Estimation of $L_{\text {eq }}$ on the assumption of Rayleigh distribution.

\begin{tabular}{|c|c|c|c|c|c|c|}
\hline \multirow[t]{2}{*}{ Equations } & \multirow[t]{2}{*}{ Noises } & \multirow{2}{*}{$\begin{array}{l}\text { Corr. } \\
\text { coef. }\end{array}$} & \multicolumn{3}{|c|}{$\begin{array}{c}\text { Differences of } L_{\mathrm{eq}} \\
\text { (measured)-(estimated) }\end{array}$} & \multirow{2}{*}{$\begin{array}{c}\text { Scale } \\
\text { parameter }\end{array}$} \\
\hline & & & Mean & Ranges & s.e. & \\
\hline \multirow{7}{*}{ Eq. $(11-1)$} & Noise 1 & 0.982 & 2.1 & $0.2 \sim 4.1$ & 0.6 & $2.9 \sim 11.0$ \\
\hline & Noise 2 & 0.979 & 2.1 & $-0.4 \sim 9.3$ & 0.8 & $4.6 \sim 16.6$ \\
\hline & Noise 3 & 0.972 & 0.7 & $0.1 \sim 1.9$ & 0.7 & $3.0 \sim 6.0$ \\
\hline & Noise 4 & 0.979 & 0.4 & $-1.6 \sim 5.3$ & 0.9 & $2.5 \sim 14.6$ \\
\hline & Noise 5 & 0.976 & 0.5 & $-0.8 \sim 3.0$ & 0.6 & $1.9 \sim 9.4$ \\
\hline & Noise 6 & 0.951 & 5.8 & $1.1 \sim 10.2$ & 1.6 & $3.8 \sim 17.8$ \\
\hline & Noise 7 & 0.983 & 3.9 & $0.3 \sim 6.7$ & 0.8 & $4.4 \sim 15.4$ \\
\hline \multirow{7}{*}{ Eq. (11-2) } & Noise 1 & 0.993 & 1.0 & $0.1 \sim 2.1$ & 0.5 & \\
\hline & Noise 2 & 0.996 & 0.5 & $-0.9 \sim 1.5$ & 0.5 & \\
\hline & Noise 3 & 0.970 & 1.1 & $0.3 \sim 2.1$ & 0.5 & \\
\hline & Noise 4 & 0.984 & 0.4 & $-2.6 \sim 3.0$ & 1.1 & \\
\hline & Noise 5 & 0.982 & 0.9 & $-0.4 \sim 2.6$ & 0.6 & \\
\hline & Noise 6 & 0.969 & 1.0 & $-1.1 \sim 3.2$ & 0.8 & \\
\hline & Noise 7 & 0.996 & -0.2 & $-1.7 \sim 1.7$ & 0.6 & \\
\hline \multirow{7}{*}{ Eq. (10) } & Noise 1 & 0.990 & 2.2 & $1.5 \sim 3.4$ & 0.5 & \\
\hline & Noise 2 & 0.989 & 1.2 & $-3.5 \sim 2.4$ & 1.1 & \\
\hline & Noise 3 & 0.973 & 1.8 & $1.3 \sim 3.1$ & 0.5 & \\
\hline & Noise 4 & 0.981 & 1.0 & $-3.1 \sim 3.7$ & 1.2 & \\
\hline & Noise 5 & 0.982 & 1.6 & $0.4 \sim 3.0$ & 0.5 & \\
\hline & Noise 6 & 0.968 & 0.4 & $-6.8 \sim 4.6$ & 2.2 & \\
\hline & Noise 7 & 0.987 & -0.3 & $-4.1 \sim 2.9$ & 1.3 & \\
\hline
\end{tabular}

Corr. coef.: correlation coefficients for regressions between $L_{\mathrm{eq}}$ measured and $L_{\mathrm{eq}}$ estimated.

cars on the road was calculated one by one for 300 meters along the road and each of noise levels was calculated at intervals of one second. Noise 7 was calculated on assumption of the normal distribution of noise levels. Table 1 shows characteristics of these noises used for estimating $L_{\mathrm{eq}}$ in this study.

Table 2 shows the results of $L_{\text {eq }}$ estimated on the assumption of Weibull distribution. Table 2 demonstrates that Weibull distribution is very effective to estimate $L_{\text {eq }}$, because the correlations are very high (statistically significant at $p<0.01$ ), the standard errors are smaller than $1.5 \mathrm{~dB}(\mathrm{~A})$, and the mean differences are very small between $L_{\mathrm{eq}}$ measured and $L_{\text {eq }}$ estimated. But some cases of Noise 4 and Noise 6 showed rather large differences between $L_{\text {eq }}$ measured and $L_{\text {eq }}$ estimated.

Table 3 shows the results of $L_{\text {eq }}$ estimated on the assumption of Rayleigh distribution. Table 3 illustrates that the Eq. (11-2) is better than the Eq. (10) or the Eq. (11-1) for estimation of $L_{\text {eq }}$. Errors of estimating $L_{\mathrm{eq}}$ on the assumption of Rayleigh distribution with Eq. (11-2) were larger a little than those assumed on Weibull distribution (see Table 2 and Table 3).

\section{DISCUSSION}

As for community noise, Nishinomiya ${ }^{8)}$ and Mori et al.9) have reported the usefulness of Weibull distribution for estimating $L_{\mathrm{eq}}$ of community noise. In this study, the usefulness of Weibull distribution or Rayleigh distribution was also found in Table 3 to estimate $L_{\text {eq }}$ from $L_{5}, L_{50}$ and $L_{95}$, not only for community noise but also for traffic noise. But the limitation of both assumptions has not been clear in the previous researches. ${ }^{8,9)}$

By the way, Table 4 shows the typical examples with the larger errors of estimating $L_{\text {eq }}$ than $1.5 \mathrm{~dB}$ (A). It was suggested in Table 4 that the assumption of Rayleigh distribution for estimating $L_{\text {eq }}$ was not adequate especially in such cases as distributions of noise levels were largely skewed to lower levels or higher levels of noises and that the assumption of Weibull distribution for estimating $L_{\text {eq }}$ was also inadequate to estimation of $L_{\mathrm{eq}}$ in such cases of the noise levels as yielding to the exponential distribution. Nevertheless, the assumption of Weibull 


\section{T. YOSHIDA: ESTIMATION OF $L_{\text {eq }}$ OF VARIOUS NOISES}

Table 4 Examples with larger errors than $1.5 \mathrm{~dB}(\mathrm{~A})$.

\begin{tabular}{|c|c|c|c|c|c|c|c|c|c|}
\hline \multirow{2}{*}{ Noise } & \multicolumn{2}{|c|}{ Differences } & \multicolumn{3}{|c|}{ Parameters } & \multicolumn{4}{|c|}{ Noise levels } \\
\hline & $\left(R_{2}\right)$ & $(W)$ & $k$ & $b$ & $s$ & $L_{95}$ & $L_{50}$ & $L_{5}$ & $L_{\mathrm{eq}}$ \\
\hline Noise 4 & -2.6 & -4.3 & 0.71 & 4.89 & 9.70 & 43 & 46 & 66 & 57.3 \\
\hline Noise 7 & -1.7 & 0.3 & 1.85 & 15.73 & 15.36 & 45 & 60 & 76 & 68.4 \\
\hline Noise 7 & -1.6 & 0.4 & 1.70 & 15.09 & 14.37 & 46 & 60 & 76 & 68.9 \\
\hline Noise 4 & -1.5 & -3.7 & 0.96 & 10.96 & 14.59 & 45 & 52 & 78 & 70.6 \\
\hline Noise 7 & -1.5 & 0.4 & 1.94 & 15.21 & 15.29 & 46 & 63 & 77 & 69.4 \\
\hline Noise 4 & 2.2 & 3.7 & 0.91 & 3.62 & 5.16 & 47 & 49 & 59 & 56.2 \\
\hline Noise 4 & 3.0 & 2.5 & 1.54 & 4.14 & 3.97 & 52 & 55 & 61 & 58.8 \\
\hline Noise 3 & 2.1 & 0.2 & 4.75 & 10.79 & 5.03 & 57 & 63 & 67 & 63.9 \\
\hline Noise 1 & 2.1 & 0.4 & 4.06 & 12.11 & 5.96 & 70 & 77 & 82 & 77.8 \\
\hline Noise 6 & 3.2 & 0.2 & 6.98 & 13.69 & 5.92 & 51 & 59 & 63 & 59.8 \\
\hline Noise 6 & 3.2 & 0.2 & 7.77 & 18.54 & 8.01 & 44 & 55 & 58 & 55.2 \\
\hline Noise 6 & 2.7 & -0.1 & 8.32 & 19.40 & 8.43 & 43 & 55 & 58 & 54.7 \\
\hline Noise 6 & 2.8 & 0.2 & 6.77 & 17.23 & 7.46 & 44 & 54 & 58 & 54.6 \\
\hline
\end{tabular}

Differences are shown as ( $L_{\mathrm{eq}}$ measured $)-\left(L_{\mathrm{eq}}\right.$ estimated).

$\left(R_{2}\right)$ : Estimation by Eq. (11-2). (W): Estimation by Eq. (10).

distribution was very useful to estimate $L_{\mathrm{eq}}$ of community noise or road traffic noise in almost cases except some special cases, although those calculations were very complicated and difficult for estimating $L_{\mathrm{eq}}$. The assumption of Rayleigh distribution was also useful with permitting about $1.5 \mathrm{~dB}(\mathrm{~A})$ except some special cases, and those calculations were easy for estimating $L_{\text {eq }}$.

\section{REFERENCES}

1) S. Yamaguchi and Y. Kato, "A statistical consideration of relation between $L_{50}$ and $L_{\mathrm{eq}}$ for random noise fluctuation," J. Acoust. Soc. Jpn. (J) 44, 515518 (1988) (in Japanese).

2) M. Ohta, K. Nakamura, and A. Ikuta, "A general theory for estimating the road traffic noise based on the compound stochastic process model and its experiment," J. Acoust. Soc. Jpn. (J) 37, 437-445 (1981) (in Japanese).

3) K. Takagi, K. Hiramatsu, and T. Yamamoto, "Investigations on road traffic noise based on an exponentially distributed vehicles model-Single line flow of vehicles with same acoustic power," J. Sound Vib. 36, 417-431 (1975).

4) M. Ohta and Y. Mitani, "A statistical analysis for estimating the noise level probability distribution from $L_{e q}$ and $L_{v}$ noise levels," J. Acoust. Soc. Jpn. (E) 10, 175-180 (1989).
5) A. Nakamo, "A relation between $L_{5}, L_{50}, L_{95}$ and $L_{\text {eq }}$," J. INCE Jpn. 8, 266-269 (1984) (in Japanese).

6) M. Ishii and H. Tachibana, "Estimation of the difference between $L_{\mathrm{eq}}$ and $L_{50}$ for road traffic noise," J. Acoust. Soc. Jpn. (J) 46, 962-969 (1990) (in Japanese).

7) H. Tachibana, K. Takagi, K. Yamamoto, T. Sone, and S. Yamaguchi, "Energy-base prediction method of road traffic noise from general types of road," Report from the Research Committee of Road Trafflc Noise, Tech. Rep. Noise Acoust. Soc. Jpn., N-92-61 (1992) (in Japanese).

8) G. Nishinomiya, "Estimation of $L_{\mathrm{eq}}$ by fitting Weibull distribution for community noises," J. Acoust. Soc. Jpn. (J) 35, 562-568 (1979) (in Japanese).

9) M. Mori and H. Tsukaguchi, "Estimation of environmental noise using Weibull distribution," J. INCE Jpn. 8, 314-320 (1984) (in Japanese).

10) A. Papoulis, Probability, Random Variables, and Stochastic Processes (McGraw-Hill, New York, 1965), p. 195.

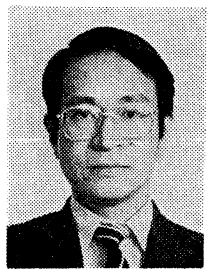

Takumasa Yoshida was born in Kobe, Japan in 1944. He joined in The Institute of Public Health (Japan) in 1968 and he has been engaged in studies about effects of noise on people. 